\title{
Isospin and a possible interpretation of the newly observed $X(1576)$
}

\author{
Feng-Kun Guo, ${ }^{1,2,5}$ and Peng-Nian Shen ${ }^{2,1,3,4}$ \\ ${ }^{1}$ Institute of High Energy Physics, Chinese Academy of Sciences, P.O.Box 918(4), Beijing 100049, China \\ ${ }^{2}$ CCAST(World Lab.), P.O.Box 8730, Beijing 100080, China \\ ${ }^{3}$ Institute of Theoretical Physics, Chinese Academy of Sciences, P.O.Box 2735, China \\ ${ }^{4}$ Center of Theoretical Nuclear Physics, National Laboratory of Heavy Ion Accelerator, Lanzhou 730000, China \\ ${ }^{5}$ Graduate University of Chinese Academy of Sciences, Beijing 100049, China
}

(Dated: August 6, 2018)

\begin{abstract}
Recently, the BES collaboration observed a broad resonant structure $X(1576)$ with a large width being around $800 \mathrm{MeV}$ and assigned its $J^{P C}$ number to $1^{--}$. We show that the isospin of this resonant structure should be assigned to 1 . This state might be a molecule state or a tetraquark state. We study the consequences of a possible $K^{*}(892)-\bar{\kappa}$ molecular interpretation. In this scenario, the broad width can easily be understood. By using the data of $B\left(J / \psi \rightarrow X \pi^{0}\right) \cdot B\left(X \rightarrow K^{+} K^{-}\right)$, the branching ratios $B\left(J / \psi \rightarrow X \pi^{0}\right) \cdot B\left(X \rightarrow \pi^{+} \pi^{-}\right)$and $B\left(J / \psi \rightarrow X \pi^{0}\right) \cdot B\left(X \rightarrow K^{+} K^{-} \pi^{+} \pi^{-}\right)$ are further estimated in this molecular state scenario. It is shown that the $X \rightarrow \pi^{+} \pi^{-}$decay mode should have a much larger branching ratio than the $X \rightarrow K^{+} K^{-}$decay mode has. As a consequence, this resonant structure should also be seen in the $J / \psi \rightarrow \pi^{+} \pi^{-} \pi^{0}$ and $J / \psi \rightarrow K^{+} K^{-} \pi^{+} \pi^{-} \pi^{0}$ processes, especially in the former process. Carefully searching this resonant structure in the $J / \psi \rightarrow$ $\pi^{+} \pi^{-} \pi^{0}$ and $J / \psi \rightarrow K^{+} K^{-} \pi^{+} \pi^{-} \pi^{0}$ decays should be important for understanding the structure of $X(1567)$.
\end{abstract}

PACS numbers: 12.39.Mk, 13.25.-k

Recently, the BES collaboration analyzed the $J / \psi \rightarrow$ $K^{+} K^{-} \pi^{0}$ decay, and found a broad resonant structure in the $K^{+} K^{-}$invariant mass spectrum. The pole position of the resonant structure is $1576_{-55-91}^{+49+98}-i 409_{-12-67}^{+11+32}$ $\mathrm{MeV}$, the $J^{P C}$ number is $1^{--}$, but its isospin has not been assigned yet [1]. They also claimed that this broad structure (refer to $X(1576)$ in the rest of the text) cannot be explained as any known mesons or their mixing states. Although the contribution from the subthreshold $\rho$ may give a significant influence on the partial wave analysis of the $J / \psi \rightarrow K \bar{K} \pi$ decay 2], the inclusion of this $\rho$ state or even other mesons cannot remove the resonant structure at $1576 \mathrm{MeV}$ and meanwhile will produce large systematic errors [1].

An important character of $X(1576)$ is that the width of about $800 \mathrm{MeV}$ is much larger than the width of any known vector mesons. The PDG data [3] show that the largest width of vector meson, for instance the width of $\rho(1450)$, is about $400 \mathrm{MeV}$. In the two-body decay process, a $J^{P}=1^{--}$vector meson could decay either into two $J^{P}=0^{-}$mesons or into one $J^{P}=0^{-}$and one $J^{P}=1^{ \pm}$(or $J^{P}=2^{+}$) mesons. In these decays, the decay width of the $1^{--}$meson would not be very large due to the $\mathrm{P}$ wave suppression in the former case and due to the phase space suppression in the later case. Therefore, it is difficult to find a proper place in the conventional $q \bar{q}$ meson spectrum for such a particle. The $X(1576)$ state cannot be a glueball state because a vector glueball consists of at least three gluons and the lattice calculation showed that the mass of the vector glueball should be about $3.8 \mathrm{GeV}$ [4. The large width of $X(1576)$ prohibits it to be assigned as a hybrid. A flux tube model calculation showed the total width of the favorable decay modes of a $1^{--}$hybrid at $2 \mathrm{GeV}$ is much smaller than $800 \mathrm{MeV}[5]$. Besides, one argued that the width of a $1^{--}$hybrid decaying into $K \bar{K}$ vanishes [ $[$ ]. It means that $X(1576)$ found in the $K^{+} K^{-}$invariant mass spectrum cannot be assigned as a vector hybrid. The width of $X(1576)$ is consistent with the argument that the width of a multi-quark state who is falling apart should be at least $500 \mathrm{MeV}[\underline{6}]$. Thus, the room left for $X(1576)$ is the tetraquark state and the meson-meson molecular state. At least, such structures should be the dominant components in $X(1576)$.

In this letter, we firstly show that the isospin of the broad structure should be 1 . Then, we study some consequences of a possible molecule configuration of the broad structure $X(1576)$, i.e. $K^{*}(892)-\bar{\kappa}$ molecule. We calculate the ratio of the widths $\Gamma\left(X \rightarrow \pi^{+} \pi^{-}\right)$: $\Gamma\left(X \rightarrow K^{+} K^{-}\right): \Gamma\left(X \rightarrow K^{+} K^{-} \pi^{+} \pi^{-}\right)$, and further estimate the branching ratios of the $J / \psi \rightarrow \pi^{+} \pi^{-} \pi^{0}$ and $J / \psi \rightarrow K^{+} K^{-} \pi^{+} \pi^{-} \pi^{0}$ decays through intermediate state $X(1576)$.

In order to determine the isospin of $X(1576)$, BES Collaboration mentioned that the $J / \psi \rightarrow K_{S} K^{ \pm} \pi^{\mp}$ decay should be studied [1]. In fact, because the isospin of the $K^{+} K^{-}$system can be either 0 or 1 , the isospin of $X(1576)$ can be figured out by examining whether the $J / \psi \rightarrow X \pi^{0}$ decay favors the isospin symmetry. Namely, if the decay violates the isospin symmetry, the isospin should be 0 , otherwise it should be 1 .

If $J / \psi \rightarrow X \pi^{0}$ decay violates the isospin symmetry, $J / \psi \rightarrow X \eta$ decay must favor the isospin symmetry, and $J / \psi \rightarrow X \pi^{0}$ decay should occur through $\pi^{0}-\eta$ mixing. Following Dashen's theorem [],$\pi^{0}-\eta$ mixing should be

$$
t_{\pi \eta}=\left\langle\pi^{0}|\mathcal{H}| \eta\right\rangle=-0.003 \mathrm{GeV}^{2} .
$$

Then, the ratio of the coupling constants $g_{J / \psi X \eta}$ and 
$g_{J / \psi X \pi^{0}}$ is

$$
\left|\frac{g_{J / \psi X \pi^{0}}}{g_{J / \psi X \eta}}\right|=\left|\frac{t_{\pi \eta}}{m_{\pi^{0}}^{2}-m_{\eta}^{2}}\right|=0.01,
$$

and the ratio of the branching ratios $B\left(J / \psi \rightarrow X \pi^{0}\right)$ and $B(J / \psi \rightarrow X \eta)$ is

$$
R_{\pi^{0} / \eta} \equiv \frac{B\left(J / \psi \rightarrow X \pi^{0}\right)}{B(J / \psi \rightarrow X \eta)} \approx\left|\frac{g_{J / \psi X \pi^{0}}}{g_{J / \psi X \eta}}\right|^{2}=1 \times 10^{-4}
$$

On the other hand, BES Collaboration measured [1]

$$
B\left(J / \psi \rightarrow X \pi^{0}\right) B\left(X \rightarrow K^{+} K^{-}\right)=\left(8.5 \pm 0.6_{-3.6}^{+2.7}\right) \times 10^{-4} \text {. }
$$

If the isospin of $X(1576)$ could be 0 , one should have

$$
\begin{aligned}
B(J / \psi \rightarrow X \eta) B\left(X \rightarrow K^{+} K^{-}\right) \approx & \frac{1}{R_{\pi^{0} / \eta}} B\left(J / \psi \rightarrow X \pi^{0}\right) \\
& \times B\left(X \rightarrow K^{+} K^{-}\right) \\
> & 1 .
\end{aligned}
$$

Clearly, it cannot be true. Therefore, one can conclude that the isospin of $X(1576)$ should be 1 , and the observed structure should be the $\left(I, I_{3}\right)=(1,0)$ state of the iso-triplet. Although the state should be named as $\rho(1576)$ according to the nomenclature in PDG 3], we still use $X(1576)$ as its name in the rest of the letter for consistency.

Assuming the observed broad structure is a vector isovector state, we consider the possibility assigning it as a $K^{*}(892)-\bar{\kappa}$ molecule state. The evidences of the controversial $\kappa$ have been observed in the analysis of the $K \pi$ scattering phase shifts [8], the Dalitz Plot Analysis of the Decay $D^{+} \rightarrow K^{-} \pi^{+} \pi^{+}$[9], and the BES data of the $J / \psi$ decays [10, 11, 12]. Despite of much controversy about the mass and the width of $\kappa$, even the existence of $\kappa$, and of the large experimental error on the mass of $X(1576)$, in this molecular scenario, the large width of $\kappa$ could lead to a large width of $X(1576)$. Now, we estimate the decay widths of some possible two-body hadronic decays of $X(1576)$. The possible decay channels considered in this report are $X(1576)^{0} \rightarrow K^{+} K^{-}, \pi^{+} \pi^{-}, K^{+} K^{-} \pi^{+} \pi^{-}$. For these channels, the involved coupling constants are the same, so we can obtain the ratios of of the three branching fractions without information of the coupling constants. In the estimation, we take $m_{\kappa} \approx 750 \mathrm{MeV}$ and $\Gamma_{\kappa} \approx 550 \mathrm{MeV}$ which are predicted in the theoretical calculations [13]. Using the phase convention $\left|\bar{\kappa}^{0}\right\rangle=-\left|I, I_{3}\right\rangle=-|1 / 2,1 / 2\rangle$, the molecular state with $\left(I, I_{3}\right)=(1,0)$ can be written as

$$
\left|X^{0}\right\rangle=\frac{1}{\sqrt{2}}\left|\left(K^{*}(892)^{+} \kappa^{-}-K^{*}(892)^{0} \bar{\kappa}^{0}\right)_{X}\right\rangle,
$$

where the subscript $X$ denotes the $X$ state coupled by $K^{*}(892)$ and $\bar{\kappa}$.

$X(1576)$ state can decay into $K^{+} K^{-}$by exchanging non-strange meson between the $K^{*}(892)$ and $\bar{\kappa}$. Constrained by the parity and the angular momentum conservation laws, among light mesons $\pi, \sigma$ and $\rho$, only $\pi$ can be exchanged. In Figs. 1(a) and 1(b), we demonstrate the decays of the $K^{*}(892)^{+} \kappa^{-}$and $K^{*}(892)^{0} \bar{\kappa}^{0}$ components of $X(1576)$ into $K^{+} K^{-}$, respectively.

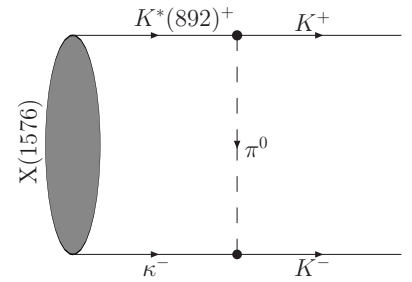

(a)

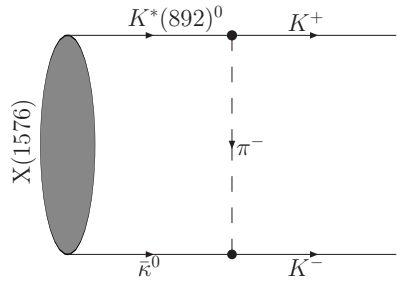

(b)
FIG. 1: Decays of $X(1576) \rightarrow K^{+} K^{-}$. (a) and (b) describe the decays of the $K^{*}(892)^{+} \kappa^{-}$and $K^{*}(892)^{0} \bar{\kappa}^{0}$ components of $X(1576)$, respectively.

The effective Lagrangian for $X(1576)-K^{*}(892)-\bar{\kappa}$ coupling can be written as

$$
\mathcal{L}_{X}=\frac{g_{1}}{\sqrt{2}}\left(\bar{\kappa} \vec{\tau} \cdot \vec{X}^{\mu} K_{\mu}^{*}+\text { H.c. }\right)
$$

where the fields of isospin multiplets are:

$$
\bar{\kappa}=\left(\kappa^{-} \bar{\kappa}^{0}\right), K_{\mu}^{*}=\left(\begin{array}{l}
K_{\mu}^{*+} \\
K_{\mu}^{*-}
\end{array}\right), \vec{\tau} \cdot \vec{X}_{\mu}=\left(\begin{array}{cc}
X_{\mu}^{0} & \sqrt{2} X_{\mu}^{-} \\
\sqrt{2} X_{\mu}^{+} & -X_{\mu}^{0}
\end{array}\right) .
$$

The $K^{*}-K-\pi$ coupling can be obtained from the $S U(3)$ symmetric Lagrangian [14]

$$
\mathcal{L}_{V P P}=\frac{i}{2} G_{V} \operatorname{Tr}\left(\left[P, \partial_{\mu} P\right] V^{\mu}\right),
$$

where $P$ and $V_{\mu}$ are $3 \times 3$ matrices, $P=\sum_{a=1}^{8} \lambda^{a} P^{a}$ and $V_{\mu}=\sum_{a=1}^{8} \lambda^{a} V_{\mu}^{a}$ with $\lambda^{a}$ being the Gell-Mann matrices. And the $\kappa-K-\pi$ coupling can be obtained from the effective Lagrangian [15]

$$
\mathcal{L}_{\kappa K \pi}=-\frac{1}{\sqrt{2}} g_{\kappa K \pi}\left(\partial_{\mu} \bar{K} \vec{\tau} \cdot \partial^{\mu} \vec{\pi} \kappa+\text { H.c. }\right) \text {. }
$$

It should be mentioned that these Lagrangians were used and tested by the others in the meson-meson scattering and the meson decay processes [14]. Because near the threshold of a particular channel, the interaction between the particles in the channel is rather weak, the t-channel contribution would dominate and the weakly bound approximation would be a good approximation, namely this loosely bound system would easily break up to the free particles of which the corresponding component of the system was composed. We also enforce a bound state condition to the system by restricting the invariant mass in the decay channel being equivalent to the mass of the decay state. Thus, near threshold a meson-meson interaction model would be appropriate for a loosely bound system. 
With these effective Lagrangians and the isospin symmetry, one finds

$$
\mathcal{M}\left(X \rightarrow K^{+} K^{-}\right)_{b}=-2 \mathcal{M}\left(X \rightarrow K^{+} K^{-}\right)_{a},
$$

where the subscripts $a$ and $b$ denote Fig. 1(a) and 1(b), respectively. Then, the total decay amplitude of the $X(1576) \rightarrow K^{+} K^{-}$process reads

$$
\mathcal{M}_{K K} \equiv \mathcal{M}\left(X \rightarrow K^{+} K^{-}\right)=-\mathcal{M}\left(X \rightarrow K^{+} K^{-}\right)_{a} .
$$

In calculating the partial decay width of the $X(1576) \rightarrow$ $K^{+} K^{-}$process, a 3-meson loop, containing $K^{*}, \kappa$ and $\pi$ propagators, is involved in the transition amplitude (see Fig. (1). In order to simplify the calculation, the on-shell approximation for the $K^{*}$ propagator is employed in dealing with loop integration, namely, the denominator of the $K^{*}$ propagator is replaced by $(-i \pi) \delta\left(k^{2}-m_{K^{*}}^{2}\right)$. A similar replacement for the $\kappa$ propagator is also performed. Then, the decay amplitude can be re-written as

$$
\begin{aligned}
\mathcal{M}_{K K}= & \frac{\pi^{2}}{2} g_{1} g_{K^{*} K \pi} g_{\kappa K \pi} \int \frac{d^{4} k}{(2 \pi)^{4}} \delta\left(k^{2}-m_{K^{*}}^{2}\right) \\
& \delta\left((p-k)^{2}-m_{\kappa}^{2}\right) p_{2} \cdot\left(k-p_{1}\right) \\
& \varepsilon(X)_{\mu} \frac{-g^{\mu \nu}+k^{\mu} k^{\nu} / m_{K^{*}}^{2}}{\left(k-p_{1}\right)^{2}-m_{\pi}^{2}}\left(2 p_{1}-k\right)_{\nu},
\end{aligned}
$$

where $g_{K^{*} K \pi}=G_{V}$, and $p, k, p_{1} p_{2}$ are 4-momenta of the $X(1576), K^{*}(892)^{+}, K^{+}$and $K^{-}$particles, respectively.

For the $X(1576) \rightarrow \pi^{+} \pi^{-}$process, $X(1576)$ can decay via $K$ exchange shown in Fig. 22 The decay amplitude

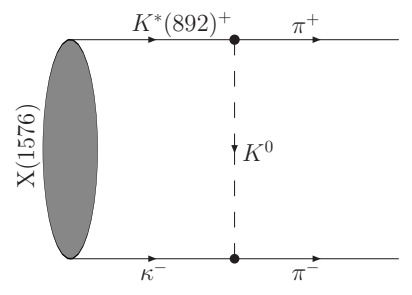

(a)

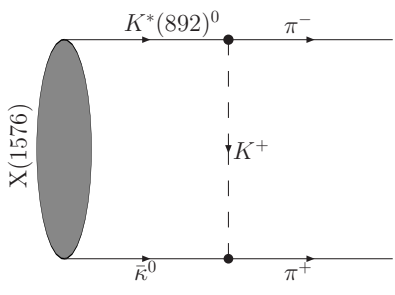

(b)
FIG. 2: Decay of $X(1576) \rightarrow \pi^{+} \pi^{-}$(a) and (b) denote the decays of the $K^{*}(892)^{+} \kappa^{-}$and $K^{*}(892)^{0} \bar{\kappa}^{0}$ components of $X(1576)$, respectively.

for Fig. 2(a) can be obtained from that for Fig. 1(a) by replacing $m_{\pi}$ with $m_{K}$

$$
\mathcal{M}\left(X \rightarrow \pi^{+} \pi^{-}\right)_{a}=-\left.2 \mathcal{M}\left(X \rightarrow K^{+} K^{-}\right)_{a}\right|_{m_{\pi} \leftrightarrow m_{K}},
$$

while the decay amplitude for Fig. 2(b) can be obtained from $\mathcal{M}\left(X \rightarrow \pi^{+} \pi^{-}\right)_{a}$ by replacing the momentum of $\pi^{+}$with the momentum of $\pi^{-}$

$$
\mathcal{M}\left(X \rightarrow \pi^{+} \pi^{-}\right)_{b}=-\left.\mathcal{M}\left(X \rightarrow \pi^{+} \pi^{-}\right)_{a}\right|_{p_{\pi^{+}} \leftrightarrow p_{\pi^{-}}} .
$$

Then, the total decay amplitude of the process $X(1576) \rightarrow \pi^{+} \pi^{-}$can be written as

$\mathcal{M}\left(X \rightarrow \pi^{+} \pi^{-}\right)=\mathcal{M}\left(X \rightarrow \pi^{+} \pi^{-}\right)_{a}+\mathcal{M}\left(X \rightarrow \pi^{+} \pi^{-}\right)_{b}$.

Since the most dominant decay channel for $K^{*}(892)$ and $\kappa$ is the $K \pi$ channel, it is interesting to study the $X(1576) \rightarrow K K \pi \pi$ decays. Because in the experiment, the charged particles in the final state are much easier to be detected than the neutral ones, we only calculate the $X(1576) \rightarrow K^{+} K^{-} \pi^{+} \pi^{-}$decay. In this decay, only the $K^{*}(892)^{0}-\bar{\kappa}^{0}$ component provides non-zero contribution. The corresponding decay diagram is shown in Fig. 3 The decay amplitude of this process reads

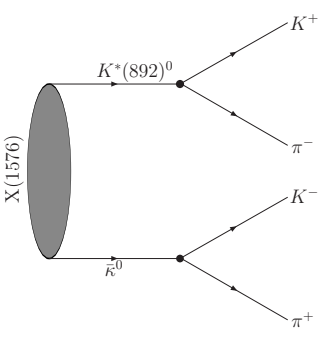

FIG. 3: Decay of $X(1576) \rightarrow K^{+} K^{-} \pi^{+} \pi^{-}$.

$$
\begin{aligned}
\mathcal{M}_{K K \pi \pi} & \equiv \mathcal{M}\left(X \rightarrow K^{+} K^{-} \pi^{+} \pi^{-}\right) \\
& =-i g_{1} g_{K^{*} K \pi} g_{\kappa K \pi} \frac{p_{3} \cdot p_{4}}{m_{34}^{2}-m_{\kappa}^{2}+i m_{\kappa} \Gamma_{\kappa}\left(m_{34}\right)} \\
& \times \frac{\varepsilon(X)_{\mu}\left(-g^{\mu \nu}+p_{12}^{\mu} p_{12}^{\nu} / m_{12}^{2}\right)\left(p_{1}-p_{2}\right)_{\nu}}{m_{12}^{2}-m_{K^{*}}^{2}+i m_{K^{*}} \Gamma_{K^{*}}\left(m_{12}\right)},(17)
\end{aligned}
$$

where $p_{1}, p_{2}, p_{3}$ and $p_{4}$ represent the 4-momenta of $K^{+}$, $\pi^{-}, K^{-}$and $\pi^{+}$, respectively. $p_{12}=p_{1}+p_{2}$ is the momentum of the $K^{+} \pi^{-}$system, and $m_{12}$ and $m_{34}$ are the invariant masses of the $K^{+} \pi^{-}$and $K^{-} \pi^{+}$systems, respectively. The energy dependent widths of $K^{*}$ and $\kappa$ are

$$
\begin{aligned}
\Gamma_{K^{*}}\left(m_{12}\right) & =\Gamma_{K^{*} 0} \frac{m_{K^{*}}^{2}\left|\vec{p}_{1}\right|^{3}}{m_{12}^{2}\left|\vec{p}_{10}\right|^{3}}, \\
\Gamma_{\kappa}\left(m_{34}\right) & =\Gamma_{\kappa 0} \frac{m_{\kappa}^{2}\left|\vec{p}_{3}\right|}{m_{34}^{2}\left|\vec{p}_{30}\right|}\left(\frac{m_{34}^{2}-m_{K}^{2}-m_{\pi}^{2}}{m_{\kappa}^{2}-m_{K}^{2}-m_{\pi}^{2}}\right)^{2},
\end{aligned}
$$

where $\Gamma_{K^{*} 0}$ and $\Gamma_{\kappa 0}$ are the decay widths of $K^{*}(892)$ and $\kappa$ at rest, respectively, their values are taken to be $\Gamma_{K^{*} 0}=51 \mathrm{MeV}$ and $\Gamma_{\kappa 0}=550 \mathrm{MeV}$, respectively,

$$
\begin{aligned}
& \left|\vec{p}_{1}\right|=\frac{1}{2 m_{12}} \sqrt{\left(m_{12}^{2}-\left(m_{K}+m_{\pi}\right)^{2}\right)\left(m_{12}^{2}-\left(m_{K}-m_{\pi}\right)^{2}\right)}, \\
& \left|\vec{p}_{3}\right|=\frac{1}{2 m_{34}} \sqrt{\left(m_{34}^{2}-\left(m_{K}+m_{\pi}\right)^{2}\right)\left(m_{34}^{2}-\left(m_{K}-m_{\pi}\right)^{2}\right)},
\end{aligned}
$$

and $\left|\vec{p}_{10}\right|$ and $\left|\vec{p}_{30}\right|$ can be obtained by replacing $m_{12}$ and $m_{34}$ with $m_{K^{*}}$ and $m_{\kappa}$, respectively. 
Then the partial widths can be calculated by using the formula [3]

$$
\Gamma=\frac{1}{2 m_{X}} \int \bar{\sum}|\mathcal{M}|^{2}(2 \pi)^{4} \delta\left(p-\sum_{i=1}^{n} p_{i}\right) \prod_{i=1}^{n} \frac{d^{3} p_{i}}{(2 \pi)^{3} 2 E_{i}},
$$

where $\bar{\sum}$ denotes the average over the polarization directions of the $X(1576)$ state.

Although the value of $g_{1}$ is not known, the ratio of the partial widths of three decay modes $X(1576) \rightarrow \pi^{+} \pi^{-}$, $X(1576) \rightarrow K^{+} K^{-}$and $X(1576) \rightarrow K^{+} K^{-} \pi^{+} \pi^{-}$can be evaluated as

$$
\begin{aligned}
\Gamma\left(X \rightarrow \pi^{+} \pi^{-}\right) & : \Gamma\left(X \rightarrow K^{+} K^{-}\right): \Gamma\left(X \rightarrow K^{+} K^{-} \pi^{+} \pi^{-}\right) \\
& \approx 19: 1: 0.18 .
\end{aligned}
$$

Whether the estimated decay width ratio of the $\pi^{+} \pi^{-}$ decay mode to the $K^{+} K^{-}$decay mode is reliable can be checked by comparing with the ratio in a naive estimation. In the later estimation, if neglecting the mass difference between $\pi$ and $K$, one can naively expect a ratio of 16 , because comparing with $\mathcal{M}\left(X \rightarrow K^{+} K^{-}\right)_{a}$, there is an extra factor of 2 in $\mathcal{M}\left(X \rightarrow \pi^{+} \pi^{-}\right)_{a}$ due to the isospin symmetry, and Fig. 22 a) and 2 (b) give the same contribution.

Note that because the dominant decay mode of the $\kappa$ is $K \pi$ and the decay amplitude of $\kappa \rightarrow K \pi$ is proportional to the coupling constant $g_{\kappa K \pi}$, the large width of the $\kappa$ requires that $g_{\kappa K \pi}$ is large. The same constant $g_{\kappa K \pi}$ appears in all of the above three partial widths, and hence it will induce a large width of the $X(1576)$.

The above decay ratio also shows that in the $K^{*}(892)$ $\bar{\kappa}$ molecular scenario, comparing with the $X(1576) \rightarrow$ $K^{+} K^{-}$decay, $X(1576) \rightarrow \pi^{+} \pi^{-}$decay is much more favorable, and the $X(1576) \rightarrow K^{+} K^{-} \pi^{+} \pi^{-}$decay is suppressed by the four-body phase space.

By using the above resultant ratio in Eq.21) and the measured branching ratio $B\left(J / \psi \rightarrow X \pi^{0}\right) B(X \rightarrow$ $K^{+} K^{-}$) [1] in Eq. (44), the following branching ratios can be estimated:

$$
B\left(J / \psi \rightarrow X \pi^{0}\right) B\left(X \rightarrow \pi^{+} \pi^{-}\right) \approx(0.8-2.2) \%
$$

and

$$
B\left(J / \psi \rightarrow X \pi^{0}\right) B\left(X \rightarrow K^{+} K^{-} \pi^{+} \pi^{-}\right) \approx(0.8-2.1) \times 10^{-4} .
$$

Comparing with the previously measured branching ra$\operatorname{tios} B\left(J / \psi \rightarrow \pi^{+} \pi^{-} \pi^{0}\right)=(1.5 \pm 0.2) \%$ and $B(J / \psi \rightarrow$ $\left.K^{+} K^{-} \pi^{+} \pi^{-} \pi^{0}\right)=(1.2 \pm 0.3) \%$ [3, 16], there is still a room for interpreting the $X(1576)$ state as a $K^{*}(892)-\bar{\kappa}$ molecular state. If this state really exists, the intermediate state $X(1576)$ should provide a dominant contribution to the decay width of $J / \psi \rightarrow \pi^{+} \pi^{-} \pi^{0}$, but not significant contribution to the decay width of $J / \psi \rightarrow$ $K^{+} K^{-} \pi^{+} \pi^{-} \pi^{0}$. Therefore, in the invariant mass spectrum of $\pi^{+} \pi^{-}$, one should observe this resonant structure even easier. Moreover, this feature can somehow be used to distinguish the structure of the $X(1576)$ state. In the $q s \bar{q} \bar{s}(q=u, d)$ tetraquark model, the $\pi \pi$ decay mode would be much suppressed [17, 18]. In the $K \bar{K}$ molecular state model, such decay modes would also be suppressed in comparison with the $K \bar{K}$ decay mode since the nonstrange decay modes will take place through $K \bar{K} \rightarrow \pi \pi$ conversion. Therefore, the relatively larger branching ratio of the non-strange decay mode is a strong signal of the $K^{*} \bar{\kappa}$ molecular model. We suggest to search the resonant structure in the $J / \psi \rightarrow \pi^{+} \pi^{-} \pi^{0}$ decay channel by using the high statistic BESII data. It is also valuable to re-analyze the $K^{+} K^{-} \pi^{+} \pi^{-}$invariant mass spectrum carefully by using the data of the $J / \psi \rightarrow K^{+} K^{-} \pi^{+} \pi^{-} \pi^{0}$ decay, although it is not so easy.

The $X$ state can also decay into other channels. For instance, $\phi \pi$ channel would not be forbidden by any symmetry and can take place through quark recombination, namely the $s$ quark in $K^{*}$ and the $\bar{s}$ anti-quark in $\bar{\kappa}$ can be combined into a $\phi$ meson, and the rest quark and anti-quark can be combined into a $\pi$ meson. The decay rate of such a channel in this scenario should further be investigated.

In summary, by utilizing the $\pi^{0}-\eta$ mixing mechanism of the isospin violated decay, we can assign the isospin of the newly observed broad structure $X(1576)$ to 1 definitely. If the observed broad structure is a physical state, it is difficult to interpret it as a conventional $q \bar{q}$ vector meson or a glueball or a hybrid state. We propose a possible $K^{*}(892)-\bar{\kappa}$ molecular state interpretation. In this scenario, the large width can be understood easily. We further estimate the decay properties of $X(1576)$. Our results show that the $X(1576) \rightarrow \pi^{+} \pi^{-}$decay mode is much more favorable than the $X(1576) \rightarrow K^{+} K^{-}$decay mode. By comparing with the $J / \psi$ decay data taken previously, this resonant structure should also appear in the $J / \psi \rightarrow \pi^{+} \pi^{-} \pi^{0}$ and $J / \psi \rightarrow K^{+} K^{-} \pi^{+} \pi^{-} \pi^{0}$ decays in this scenario. In order to confirm this state, we suggest to re-analyze the data of the $J / \psi \rightarrow \pi^{+} \pi^{-} \pi^{0}$ and $J / \psi \rightarrow K^{+} K^{-} \pi^{+} \pi^{-} \pi^{0}$ decays collected at BESII. However, there are other possible molecule and tetraquark configurations [17, 18] that will result in different predictions. The concrete consequences of other decay channels and other possible molecule configurations, such as $K^{+} K^{-}$, will be studied in the future.

\section{Acknowledgments}

We thank S. Jin and C.-P. Shen for discussion on experimental status, and thank B.-S. Zou for useful discussion. This work is partially supported by the NSFC grant Nos. 10475089, 10435080, CAS Knowledge Innovation Key-Project grant No. KJCX2SWN02 and Key Knowledge Innovation Project of IHEP, CAS (U529). 
[1] M. Ablikim, et al. (BES Collaboration), hep-ex/0606047 submitted to Phys. Rev. Lett..

[2] F.Q. Wu and B.S. Zou, Phys. Rev. D 73, 114008 (2006).

[3] S. Eidelman et al. (Particle Data Group), Phys. Lett. B 592, 1 (2004).

[4] C.J. Morningstar and M. Peardon, Phys. Rev. D 60, 034509 (1999); Y. Chen et al., Phys. Rev. D 73, 014516 (2006).

[5] N. Isgur, R. Kokoski, and J. Paton, Phys. Rev. Lett. 54, 869 (1985); P.R. Page, E.S. Swanson, and A.P. Szczepaniak, Phys. Rev. D 59, 034016 (1999).

[6] S. Capstick, P.R. Page, and W. Roberts, Phys. Lett. B 570, 185 (2003).

[7] R. Dashen, Phys. Rev. 183, 1245 (1969).

[8] D. Aston et al., Nucl. Phys. B296,253 (1988).

[9] E791 Collaboration, E. M. Aitala et al., Phys. Rev. Lett. 89, 121801 (2002).

[10] N. Wu, International Symposium on Hadron Spec- troscopy, Chiral Symmetry and Relativistic Description of Bound Systems, Tokyo, Japan, February 24-26, 2003.

[11] D. V. Bugg, Phys. Rept. 397, 257 (2004).

[12] BES Collaboration, M. Ablikim et al., Phys. Lett. B 633, 681 (2006).

[13] E.g., E. van Beveren et al., Z. Phys. C 30, 615 (1986); D.V. Bugg, Phys. Lett. B 632, 471 (2006); F.-K. Guo et al., Nucl. Phys. A773, 78 (2006); Z.Y. Zhou and H.Q. Zheng, hep-ph/0603062

[14] D. Lohse, J.W. Durso, K. Holinde, and J. Speth, Nucl. Phys. A516, 513 (1990).

[15] D. Black, A.H. Fariborz, F. Sannino, and J. Schechter, Phys. Rev. D 59, 074026 (1999).

[16] M.B.E. Franklin et al., Phys. Rev. Lett. 51, 963 (1983).

[17] M. Karliner and H.J. Lipkin, hep-ph/0607093

[18] G.-J. Ding and M.-L. Yan, hep-ph/0607253 